\title{
An efficient GAF routing protocol using an optimized weighted sum model in WSN
}

\author{
Hanane Aznaoui ${ }^{1}$, Arif Ullah' ${ }^{2}$, Said Raghay ${ }^{3}$, Layla Aziz ${ }^{4}$, Mubashir Hayat Khan ${ }^{5}$ \\ ${ }^{1,3}$ Cady Ayyad University, Faculty of Sciences and Techniques, LAMAI Laboratory, Marrakech, Morocco \\ ${ }^{4}$ FPSB-Chouaib Doukkali University \\ ${ }^{2,5}$ Faculty of Computer Science and Information Technology, Universiti Tun Hussein Onn Malaysia, Johor, Malaysia
}

\begin{tabular}{|c|c|}
\hline Article Info & ABSTRACT \\
\hline Article history: & A wireless sensor network (WSN) is composed of a large number of sensor \\
\hline Received Aug 22, 2020 & The principal tasks of nodes are gathering and transmitting data collected to \\
\hline Revised Jan 19, 2021 & the base station (BS). Consequently, the essential criteria for designing a WSN \\
\hline Accepted Feb 5, 2021 & $\begin{array}{l}\text { are the network lifetime. In this paper, we proposed an efficient geographic } \\
\text { adaptative fidelity (GAF) routing protocol for gathering data. In this system, }\end{array}$ \\
\hline Keywords: & $\begin{array}{l}\text { sensor nodes are distributed using Gaussian law, and an active leader is elected } \\
\text { for each virtual grid to reduce the energy dissipated. An optimized weighted }\end{array}$ \\
\hline Wireless sensor networks & $\begin{array}{l}\text { sum model is used to resolve this problem, where the maximum remaining } \\
\text { energy and minimum distance criteria are considered as essential criteria. }\end{array}$ \\
\hline Location-based & Moreover, routing data focuses on the transmission range for enhancing \\
\hline GAF & energy efficiency. The experimental results show that the proposed energy- \\
\hline Routing & efficient GAF produces better performance than the existing, GAF basic and \\
\hline Weighted sum model & optimized-GAF routing protocol in terms of the number of dead nodes and \\
\hline Energy-consumption & energy consumption. It is obviously proved that the proposed efficient GAF \\
\hline Dead nodes & can improve the network's lifetime. \\
\hline
\end{tabular}

This is an open access article under the $\underline{C C B Y-S A}$ license.

\section{Corresponding Author:}

Hanane Aznaoui

Universiti Cady Ayyad

Faculty des Sciences et Techniques

Laboratoire de Mathématiques, Appliquées et Informatique Marrakech, Morocco

Email: H.aznaoui@gmail.com

\section{INTRODUCTION}

A wireless sensor network (WSN) consists of a huge number of micro-sensor nodes used to supervise areas of interest, collect and transmit data to the base station (BS). Due to the small size of sensor nodes and wireless transmission process. WSN is applied and implemented in all domains especially in military applications, transport, tracking, health, and home monitoring. A WSN is composed of a large number of sensor nodes, which are randomly scattered in all over the network. Then depending on the type of applications, there is a multitude of sensors on the market, however, with this apparent diversity, they still have similar hardware architecture. The main components of sensor nodes are three units [1,2]. The sensing unit, which is responsible for collecting data from the environment, the processing unit, performs processing on the data (if necessary) and decides when and where to send them (must implement programs and different communication protocols). It is an intermediate between the sensing unit and transmission unit, and the transmission unit, which is responsible for all the data transmissions via wireless communication. Then all units are alimented by the power unit $[3,4]$. 
Sensor nodes allow collecting all types of events or information (humidity, temperature, pressure, brightness, sound, or others) and transmitting them into the base station (BS) called the Sink. The BS requests for information by sending a query in all over the network. When the node discovers the data corresponding to the query response is transmitted back to the BS [5, 6]. According to the hardware architecture of the sensor node, it is not possible in some applications to recharge or to change its battery. So that, the most critical scheme in WSN is sensor nodes energy. In addition, the transmission and reception data consume most of it, so optimizing energy consumed during routing data is essential to extend the network lifetime. Routing data is a principal function, which allows the distribution of messages from a network source node to a destination node in WSN. So that, several routing protocols are used [7, 8]. Were invented to ensure this function, are in fact categorized into three families, flat routing protocols where the base station sends queries and waits for data from nodes. Cluster routing protocols where the network is divided hierarchically in a base station, a master node called cluster-head, and a standard node. Moreover, location routing protocols where the sensor nodes are distributed in an area of interest and are knowing by their geographical position [9, 10]. Clustering and data gathering are efficient methods for saving energy in WSN. The main goal of clustering is to organize the network into clusters that communicate with a local cluster-head $(\mathrm{CH})$, and the $\mathrm{CH}$ transmits data to the base station to minimize sending signals from each node directly to the base station. Moreover, data gathering permits conserving energy by removing the redundant message by including some aggregation methods, which allows reducing the traffic data in the network and directly extending the network lifetime [11, 12]. Clustering data gathering or other methods that help to reduce the traffic in the network reflects directly how efficient the routing protocol used for communication. Its causes significant energy-conserving [13]. Figure 1 present the strcture of WSN.

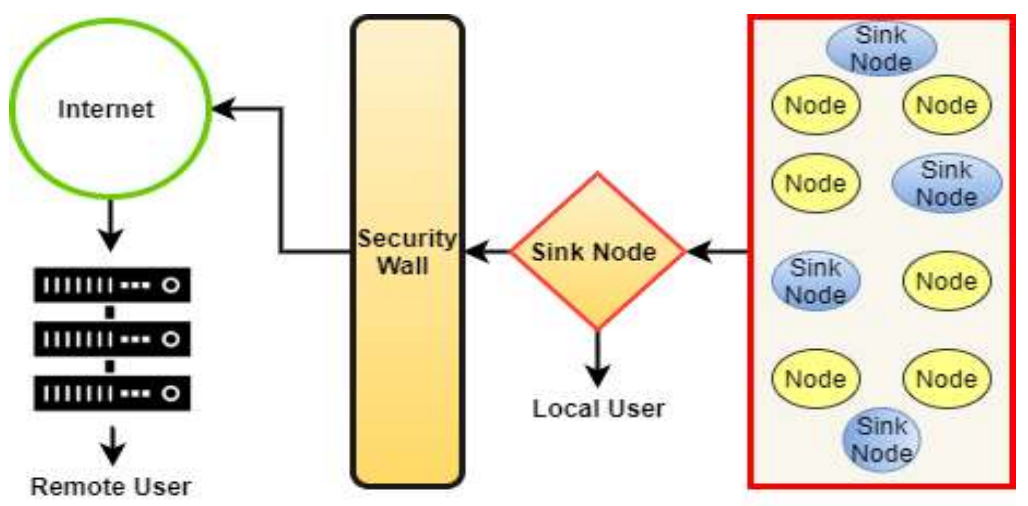

Figure 1. Structure of WSN

In this paper, a new efficient GAF protocol introduced the proposed method focuses on three enhancements. The Gaussian deployment model for nodes distribution, an optimal active leader's selection by using an optimized weighted sum model. The data routed from the different active leaders to the BS based on transmission range. The rest of the paper is organized as follows. Section 2 presents a description of the previous research that is relevant to energy-efficient data-gathering and location-based approaches. Section 3 describes our proposed efficient GAF (E-GAF) Protocol. Section 4 presents the results and discussion. Section 5 discusses the conclusions and future work.

\section{RELATED WORK}

Geographic adaptive fidelity is an energy-efficient routing protocol location-based. It was conceived initially for ad hoc networks. However, it is also applied in sensor networks. This protocol can use mobile and non-mobile nodes. Although it may also be implemented as a hierarchical architecture were the creation of clusters based on the geographic position [14]. In the GAF routing protocol, the area of interest is divided into equal virtual grids (Figure 1). Where sensor nodes use GPS (global position system) to associate each node to its grid in the area of interest. Also, a node in each grid has different states of transition as shown in Figure 2; the active state where the active node is responsible for routing data, the discovery state is used to determine the neighbors in the grid or sleeping state where the radio is turned off. In terms of packet routing, nodes in the same grid are considered equal. This equivalence help keeping one active node in each grid while the remaining nodes in the same grid are in sleeping mode. GAF also can conserve energy consumed by turning off the no 
active nodes. For that GAF routing protocol increase the energy consumed as the number of dead nodes increases, which helps to extend the network lifetime $[15,16]$.

Grid-Based coordinated routing (GBCR) proposes different schemes that allow reducing the number of grids that are essential for supporting routing during network connectivity. Authors proved that diagonal routing based on diverse side lengths of grids overtakes direct routing in populated sensor networks, taking into account transmit power and receiver sensitivity, parameters constant values such as path loss supporter, then the variable of no uniform and uniform grids [17].

Two-level GAF (TGAF) routing protocol the author suggested to overcome the problem of communication between only neighboring grids, it helps to reduce the number of a hop count to facilitate to the sensors in the same grid forwarding the data packet to a sensor of the neighbor of its adjacent grids, which permits reducing the number of nodes responsible for routing data. The main goal of TGAF based on using two levels for transmitting data and it used the highest residual energy for selecting active nodes. The authors prove that this concept help in minimizing the energy consumed and automatically extending the network lifetime [18].

Optimized-GAF is an improved version of GAF the improvement over this protocol was performed on the node transition state. This approach has also three states as the original GAF as shown in Figure 3. However, its process is executed differently where the main aim of the discovery phase is defining the sequence of nodes that will be picked as active nodes. This phase is executed once time hence, the energy consumed is minimized by the author used this technique [19]. The transition phase of the proposed technique performed as follows:

a) Discovery phase: It is based on defining a sequence of nodes chosen to play the role of active nodes. The nodes picked considering the highest remaining energy, this state performed only once time for reducing the energy consumed.

b) Active phase: After Ta (time of active node), the active node is selected among the sequence without executing the previous state.

c) Sleep phase: After Ts (time sleeping), the followed node will be chosen to be an active node.

The transition phase of the proposed technique performed as follows:

Discovery phase: It is based on defining a sequence of nodes chosen to play the role of active nodes. The nodes picked considering the highest remaining energy. This state performed only once time for reducing the energy consumed.

d) Active Phase: After Ta (time of active node), the active node is selected among the sequence without executing the previous state.

e) Sleep Phase: After Ts (time sleeping), the followed node will be chosen to be an active node.

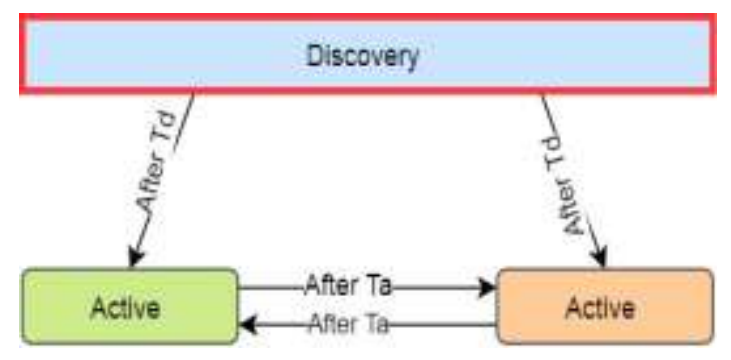

Figure 2. Transition state in GAF

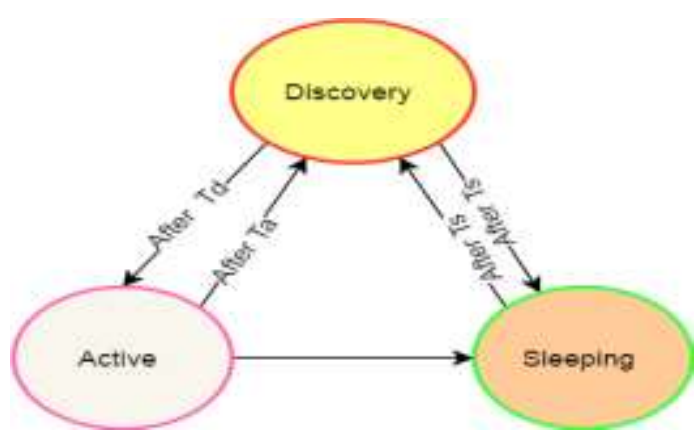

Figure 3. Transition state in optimized-GAF

Various efficient QoS routing protocols are designed to be adapted to real-time application needs. Grid-based Multipath with congestion avoidance routing protocol (GMCAR) represents one of the known routing protocols belonging to this category [20, 21]. Predictive geographical routing (GPGR) is designed for inter-vehicle communications (IVC). It exploited the map data for generating the road grid. Moreover, it generates the moving path of vehicles where its location is previously known by GPS. Thus, forecasting the road grids done in a real way instead of the forecast is blind. To evaluate the influence of the grid form, a comparative study between the square grid and hexagonal grid was performed in [22, 23]. The hexagonal form represents efficient results compared to other routing techniques in terms of energy consumed. However, it is required to handle the network overhead. On the other hand, the grid form is judged more suitable for coverage performance. Also, it is characterized by its simple implementation and clustering [24]. 
In [25], the authors proposed an energy-efficient multipath grid-based geographic routing protocol (EMGR), which is suitable for underwater wireless sensor networks (UWSN). It considers grids in 3D, where routing data have been done between grids. Besides, this new approach uses disjoints paths for achieving the routing phase. This phase uses an improved algorithm, which is based on three essential points. The first one aims at electing the most effective gateway based on two different criteria the location grid and the residual energy [26]. The second point aims to update gateways' information using a technique of memorization applied to sensor nodes. The third point is responsible for establishing disjoints paths using a packet transmission technique [27]. A simulation study proved that this collaborative approach improved energy consumed, endto-end delay, and delivery ratio significantly.

In [28], the authors designed a novel approach, which exploited the grid clustering for enhancing the network energy consumed. In the clustering phase, $\mathrm{CHs}$ are selected based on their residual energy and buffer size, and distance from a cluster member. moreover, it integrated the shortest path algorithm for improving data routing [29]. For resolving the path energy problem [30], this approach is considered a wireless portable charging device (WPCD) that followed sensor nodes to charge them when required. This is done by using the wireless power transfer (WPT).

\section{PROPOSED PROTOCOL NEW GAF}

Our proposed approach is based on three new enhancements, which will be detailed later in each part. The first one is the network model distribution using Gaussian low [31]while the basic GAF uses a random distribution, Also the basic GAF did not consider the remaining energy during active nodes selection. However, the second improvement in our case is the selection of active leaders using a strong model, which aims at taking into account two criteria of interest the maximum remaining energy and the minimum distance to the base station. Then, the last one is transmission data considering transmission range while in basic GAF a huge number of active nodes participate in routing data.

\subsection{Network model distribution}

We assume that the sensor network is composed of N nodes and the base station BS is positioned on the network area. Sensor node localization is known in our approach, we consider that sensor nodes distribution follows 2D Gaussian distribution. Hence, it is expressed as follow:

$$
f(P m, P n)=\frac{1}{2 \pi \sigma_{P m} \sigma_{P n}} e^{\left(\frac{\left(P _ { m - m _ { u } ) ^ { 2 } } { } ^ { 2 } \left(P_{\left.n-m_{v}\right)^{2}}{ }^{2}\right.\right.}{2 \sigma_{P_{m}}{ }^{2}}+\frac{\sigma_{P n}{ }^{2}}{2 \sigma^{2}}\right.}
$$

Where, (mu,nv) represents the point, $\sigma_{P m}$ and $\sigma_{P n}$ represents the deviation for $\mathrm{P}_{\mathrm{m}}$ and $\mathrm{P}_{\mathrm{n}}$ dimensions. The deployed point is considered as the central point of the grid. $\left(m_{i}=n_{i}=0\right)$. Hence, The Gaussian distribution can be expressed as:

$$
f(P m, P n)=\frac{1}{2 \pi \sigma_{P m} \sigma_{P n}} e^{-\left(\frac{\left(P_{m}\right)^{2}}{2 \sigma_{P m}{ }^{2}}+\frac{\left(P_{n}\right)^{2}}{2 \sigma_{P_{n}}{ }^{2}}\right)}
$$

Each node senses the traffic pattern about its data, and a BS is responsible for gathering the data periodically $[32,33]$.

\subsection{Active nodes selection}

Due to the main task of data routing attributed to active nodes. Its selection represents a critical issue in the basic routing protocol GAF. In this work, we consider a strong model for selecting such nodes where two criteria are considered: residual energy and distance from the sink.

\subsection{Weighted sum approach}

For multi-objective optimization and in the theory of decision making, the most popular method of multi-criteria analysis is the weighted sum method (WSM), which is considered as the simplest one that allows evaluating alternatives with taking into account criteria of interest. Where the method changes multiple objectives into an aggregated objective function by multiplying each objective function by a weighting factor and summing up all contributors. It supposes that a given problem is presented on $\mathrm{m}$ alternatives and $\mathrm{n}$ decision criteria. It helps to select the optimal alternative between others considering the criteria of decision and mathematically defined as $[34,35]$. 


$$
A_{i}=\sum_{j=1}^{n} w_{j} a_{i j}, \text { for } i=1,2,3, \ldots, m
$$

Where $\mathrm{Ai}$ is the total importance of alternative, $\mathrm{Wj}$ describes the weight of the criterion $\mathrm{Cj}$, then aij is the performance value of alternative $\mathrm{Ai}$ when it is evaluated in terms of criterion $\mathrm{Cj}$. The weighted sum method, mentioned above, is a great mathematical tool, especially in our case, because our model is based on two parameters, the energy $\mathrm{E}$ and the distance $\mathrm{d}$, so it would be written as a linear combination similar to.

$$
W 1 \frac{E}{E n}+W 2 \frac{d}{d m}
$$

Where w1 and w2 are, for the instant, are two positive constants, with $0<(w 1+w 2) \leq 1$, but of course, while the energy is maximal and the distance is minimal. E0 represents the initial energy and dm is the maximum distance between the node and the base station. In the majority of previous works, related to the decision-making problems, the weights are exploited arbitrarily. But, in our case, there are chosen based on stochastic optimization. That means that our work depends on random variables instead of normal ones [36, 37].

The dilemma is in the choice of those two constants w1 and w2, our idea is to relook for those two as the probability of two random variables X1 and X2, follow the Gaussian or normal distribution, we note:

$X i-N(\mu \delta)$, for $\mathrm{i}=1,2$, Where $\mu$ is the mean (usually taken as 0 ) and $\sigma 2$ is the variance, while the event is transferring the data depending on the parameters we have, so of course, the probability of the event is 1 , and the probability of choosing the energy as caliber is w1 and vice versa for the distance with its probability w2. One chooses this distribution because it is often used in the natural and social sciences to represent realvalued random [38].

\subsection{Proposed selection model}

According to (3), our model can be written as follow:

$$
\min \left[E(W 1, W 2)=\left(W 1 \frac{E}{E 0}+W 2 \frac{d}{d m}\right)\right]
$$

Where $\mathrm{C}$ is the constraint of the problem, described as:

$$
C=\left\{\begin{array}{l}
W 1+W 2=1 \text { and } W 1 \geq W 2 \\
\text { and } W 1, W 2 \in[a, b] \subset[0,1]
\end{array}\right\}
$$

We can rewrite our model as follow:

$$
\left.\mathrm{E}\left(\mathrm{w}_{1}\right)=E(W 1)=W 1\left(\frac{E}{E o}-\frac{d}{d m}\right)+\frac{d}{d m}\right)
$$

and that $W 1+W 2=1$ rewritten as:

We remark that our functional E can depend on one parameter (w1 for example), the problem can be

$$
E w 1=\alpha w 1+\beta
$$

Where:

$$
\begin{aligned}
& \alpha=\frac{E}{E o}-\frac{d}{d m} \\
& \beta=\frac{d}{d m}
\end{aligned}
$$

Hence, $\mathrm{E}$ is an affine function, $\alpha$ and $\beta$ don't depend on w1. We studied the different cases of the value of $\alpha$ : If $\alpha>0, E$ is an increasing function, it exists a point $\tau$, which verified: 
$E(x)>E(\gamma), \forall x \varepsilon R^{+}$So the minimum is $r, w_{1}=r$ and $w_{2}=1-r$.

Otherwise, $\alpha<0, E$ will not have a minimum in our case, because it doesn't have any sense. In fact, we found the condition $\left(E<\frac{d}{d_{m}} E_{0}\right)$ and we didn't need nodes whose energy is very low compared to d.

To overcome this problem, we added, mentioned above, a condition in the constraint, to minimize in every compact $\left[a_{1} b_{1}\right]\left[a_{1} b_{2}\right]$ of $[01] \chi[01]$

\subsection{Data routing phase}

The last phase aims at establishing a routing table for each active leader. Initially, each active leader broadcasts a HELLO message, which informs the remaining nodes about its ID and its position from the sink and the grid ID and its energy level. Then, each active leader establishes its routing table based on the transmission range. Hence, each routing table contains the active leaders, which belong to the transmission range. Consequently, each active leader can communicate its data to the active leaders belonging to the newly established table. To reduce the energy consumed by active leaders, we consider the transmission range as an important criterion for routing data efficiently. Our protocol checks if the sink belongs to its transmission range, if it is the case, it delivers data directly to the sink. However, if it is not the case, the current active leader looks for the best active leader among its routing table members. It selects an active leader as a relay, taking into account the minimal distance from the sink. The process is repeated until the data reach the sink.

\subsection{Algorithm description}

The implementation of our new proposed GAF can be divided into 4 parts. The virtual grid partition, the sensor nodes distribution using Gaussian low, the selection of active leaders using an efficient selection model described above where we improve the multi-criteria decision model weighted sum using the stochastic optimization. Then, the last phase consists of routing data through the previously selected leaders or directly to the sink based on transmission range. The first and the second steps execute only once time, while the last two steps would be executed periodically with the change of active leaders in each grid. The following algorithm describes the fourth phases:

Step 1: Target region division on virtual grids.

Step 2: Network Model Distribution phase

This initial step aims at distributing the sensor nodes using the Gaussian model as described in (1).

Step 3: Selection of Active Leaders

In this step, our approach consists of electing the most efficient sensor nodes as active leaders in each grid, using an effective decision model: Weighted sum. The main improvement of this model is determining the model weights using stochastic optimization.

Step 4: Data Routing

In this step, the packets are transmitted using a new process, which is based on the transmission range. When the sink belongs to the transmission range of the active leader, the sensed packets are routed directly to the sink. Otherwise, the current active leader elects the most efficient member from its routing table members for transmitting data to this selected element as a relay. Then, the process is repeated until the sink receives the sensed packets.

Step 5: Energy model

The energy consumption system used in this model just measured the node sending and receiving and ignores those messages generated by different usage of device and storage in the system. The node energy consumption is measured from the distance of square $\mathrm{d} / \mathrm{d}$.

$$
\begin{gathered}
\operatorname{ETx}(k, d)=\text { Eele }(k)+\operatorname{Eamp}(K, D) \\
=\left\{\begin{array}{c}
k \times \text { Eele }+k \times \varepsilon f s \times d 2, d \leq d o \\
k \times \text { Eele }+k \times \text { Emp } \times d 4, d \leq d o
\end{array}\right\} \\
\text { ERx }=\text { Eele }(k)
\end{gathered}
$$




\section{SIMULATION AND ANALYSIS}

\subsection{Simulations parameters}

To measure the performance of our proposed work, we compare it with two previously proposed approaches: basic GAF and its enhanced version Optimized GAF. To make this study, we consider different scenarios based on varying the number of nodes. The following Table 1 shows the simulation parameters used.

Table 1. Simulation parameters

\begin{tabular}{lc}
\hline \multicolumn{1}{c}{ Parameters } & Values \\
\hline Number of sensors (num) & $100,150,200,250,300,350,400,450,500$ \\
Target Region & $400 * 400$ \\
Number of Grid & $8 * 8$ \\
Transmission range (m) & 100 \\
packets size (bits) & 1000 \\
Electronic energy (nJ/bit) & 50 \\
Transmitter amplifier & 100 \\
(pJ/bit/m2) & \\
Transmission delay (sec) & 0.01 \\
Reception delay (sec) & 0.05 \\
\hline
\end{tabular}

\subsection{Energy consumption}

Energy consumption is proportional to the distance and the number of relays exploited for routing sensed data from the active leaders to the sink. Hence, the enhancements performed over the basic GAF related to the distance and the number of the selected relays improve significantly the energy consumed as shown in Figures 4 and 5. Our approach depicts low values of the energy consumed because it is based on a strong model, which considers various criteria. Moreover, it considers an efficient method based on the transmission range and the minimal distance for selecting the active leaders, which will play the role of relays to route data effectively. Consequently, our proposed approach optimizes the number of relays and the distance between the active leaders and sink, which allows decreasing the amount of the energy consumed by $54 \%$ compared to OGAF and 70\% compared to the basic GAF. However, the basic GAF shows the highest values of the energy consumed because the data packets travel on all neighboring grids, which exhausts more energy. GAF protocol consumes more energy even if only one active node is selected in each grid. The energy reduced in each grid by the activation of one node is drained in data routing while no technique is exploited for controlling the distance between grids and the sink. Optimized GAF presents a medium amount of energy consumed because it uses a new principle for transiting GAF states where the discovery phase is executed only once. However, it routes data on all neighboring grids, which increases the amount of energy compared to our approach.

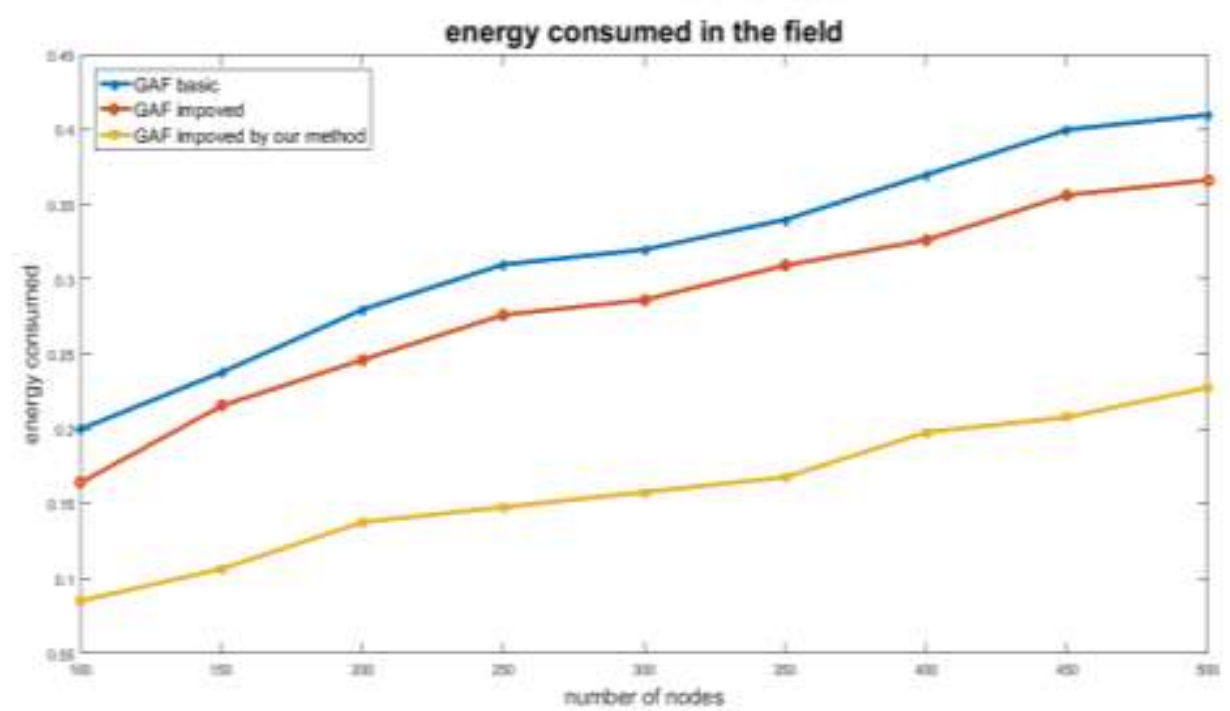

Figure 4. Comparison of energy consumed in different number of nodes 


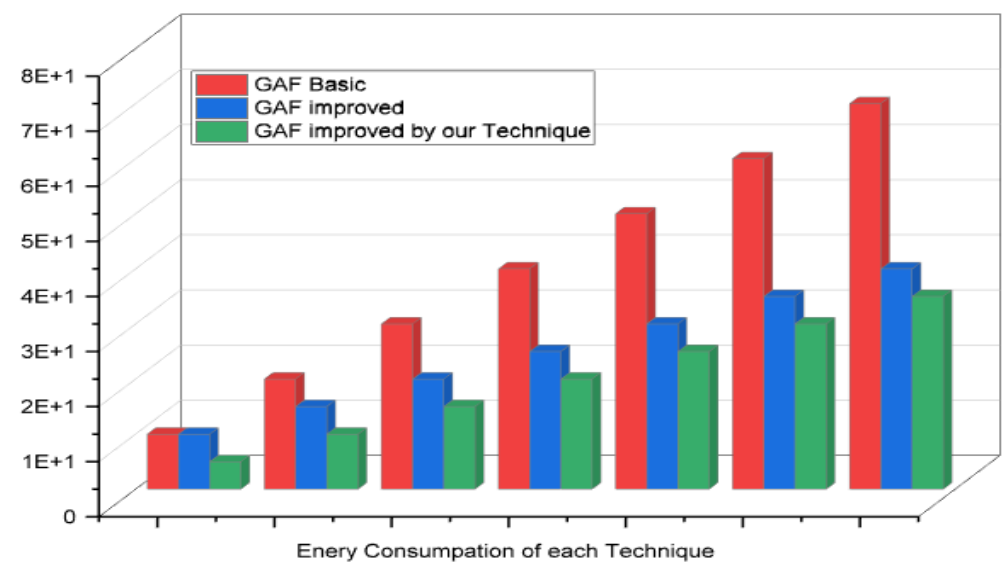

Figure 5. Comparison of energy consumed presentation

\subsection{Network lifetime}

Figures 6 and 7 depict the number of alive nodes among the three protocols. Our approach shows the highest number of living nodes comparatively to the three remaining protocols. It decreased the number of dead nodes by $40 \%$ compared to GAF and $43 \%$ compared to OGAF. The balance of energy is related to the time of the first dead node and that of the last dead node. The period is more shorten means that the energy is more balanced over the entire network and hence the total energy is balanced effectively. Therefore, it can be concluded from Figures 6 and 7 that our approach does not present only the highest number of alive nodes but an efficient improvement over the energy consumed by all nodes, which permits us to consider our approach as the most efficient in terms of energy consumed among all network nodes. From the results depicted in Figure 6, we can observe that Optimized GAF shows the highest values compared to the basic GAF due to the enhancement performed in the discovery phase. This phase consumed less energy because it saves this energy amount of each round in comparison to that in the GAF protocol.

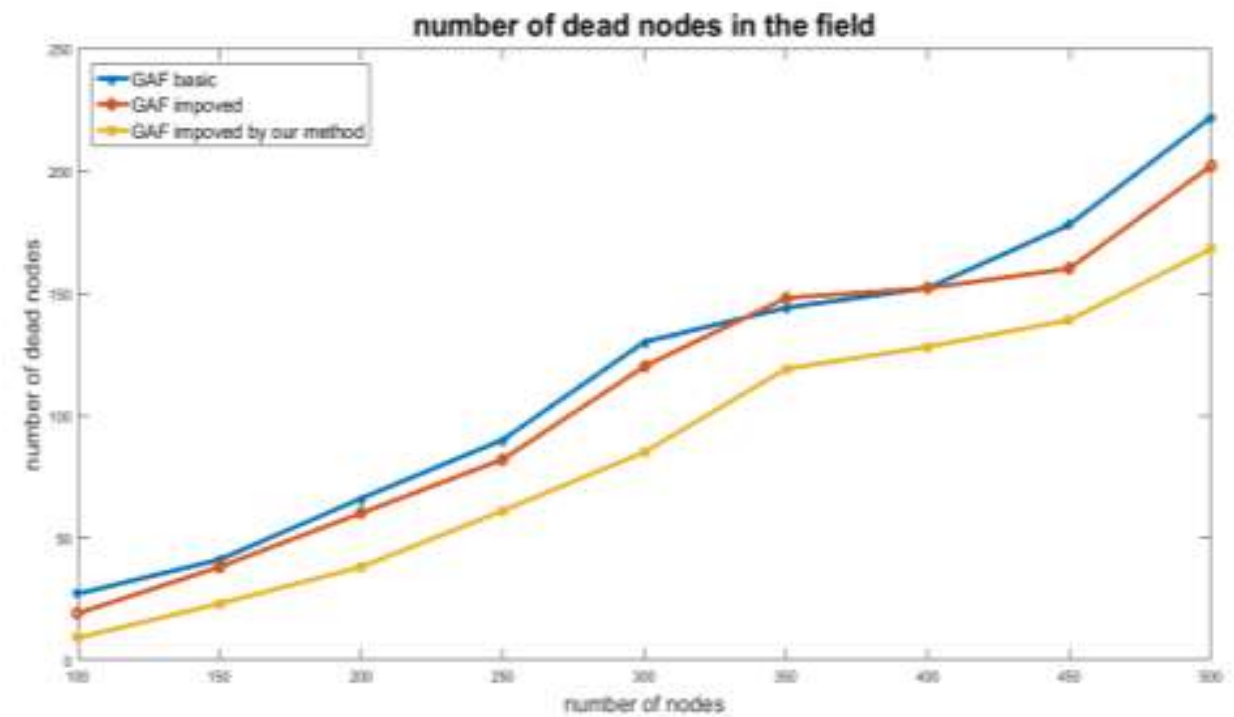

Figure 6. Comparison graph of the number of dead nodes 


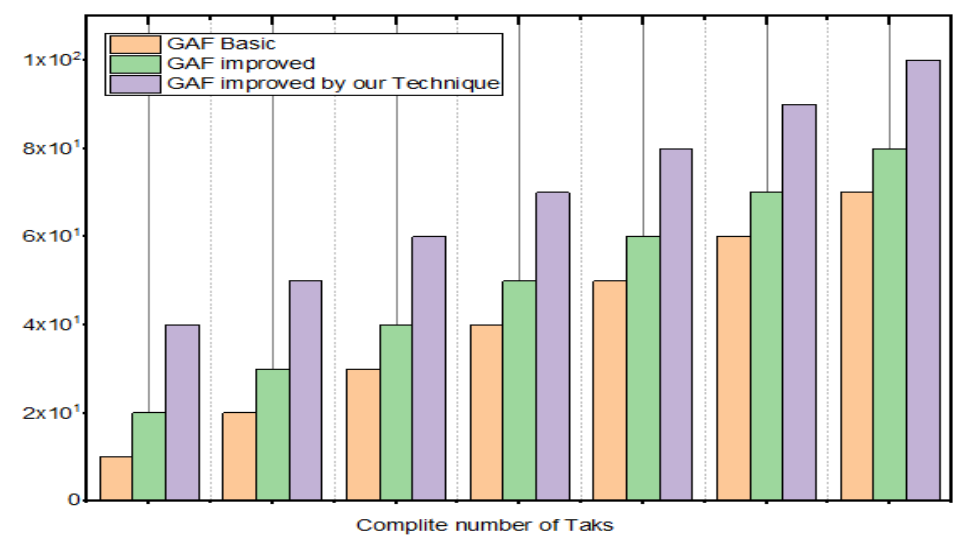

Figure 7. Number of dead nodes

\subsection{Delay}

Packet delay is proportional to the number of relays and the distance separating grids from the sink in data routing. Figures 8 and 9 depict the packet delay of the three approaches; we can observe that our approach enhances significantly the packet delay compared to the remaining protocols. This is due to the enhancements performed over the basic GAF where a strong model is exploited for selecting the active leaders. Moreover, an effective method is used for routing data diagonally based on the transmission range of active leaders and their distance from the sink. The use of an optimized number of relays and distance between grids and the sink allows reducing the packet delay by $39 \%$ and $42 \%$ compared to GAF and OGAF respectively. However, the optimized GAF and the basic GAF depict an increased amount of packet delay, because data are delivered over all neighboring active nodes. Hence, the data packet takes more time for reaching the sink node.

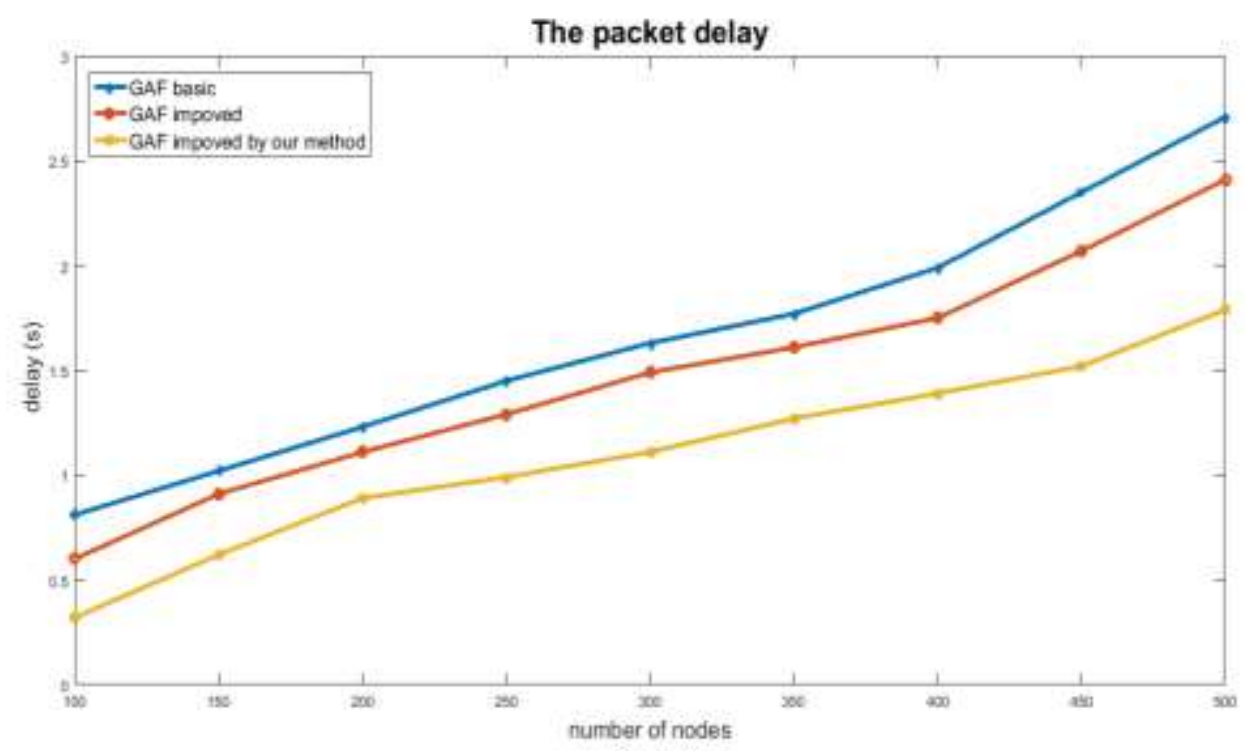

Figure 8. Comparison graph of Packet delay and different number nodes 


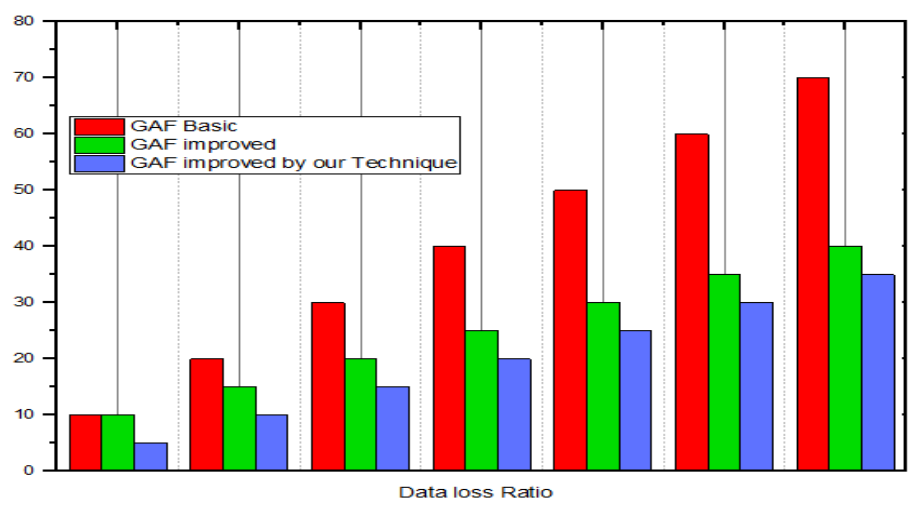

Figure 9. Comparison graph of packet delay and different number nodes

\section{CONCLUSION}

In WSN, the main problem is the higher energy consumed during communication, which causes a global death of the entire network. The main solution to overcome this problem is the use of an efficient solution to help to prolong the network lifetime. This solution reached by using different approaches such as an optimizing distribution of sensor nodes, efficient routing protocol, an optimum selection of active nodes, reducing the hop count, and so on. On the other hand, even though GAF used energy saving due to the use of one active node in each grid, and by turning off unnecessary sensors. However, GAF is still limited in terms of energy consumed because due to some drawbacks. Like the way of allowing communication between just neighboring grids, and the way of selection of active nodes without taking into account the remaining energy of nodes. For that, in this paper, a new efficient GAF routing protocol was presented to improve the energy usage, comparatively to the basic GAF, and optimized GAF protocol. Our idea focuses on the distribution of sensor nodes using the Gaussian law, the election of the active leader for each virtual grid, to reduce the energy dissipated using an optimized weighted sum model. Two parameters are considered as the main criteria: the maximum remaining energy and the minimum distance. The simulation results prove that the proposed E-GAF produces better performance than the existing GAF basic and optimized-GAF routing protocol in terms of the number of dead nodes, energy consumption, and packet delay. It is proved that the proposed EE-GAF improved the network's lifetime.

\section{REFERENCES}

[1] Baseer, S., \& Umar, S. "Role of cooperation in energy minimization in visual sensor network," In 2016 Sixth International Conference on Innovative Computing Technology (INTECH), pp. 447-452, IEEE, 2016.

[2] L. Aziz, S. Raghay, H. Aznaoui, and A. Jamali, "A new approach based on a genetic algorithm and an agent cluster head to optimize energy in Wireless Sensor Networks," 2016 International Conference on Information Technology for Organizations Development (IT4OD), Fez, Morocco, 2016, pp. 1-5, 2016.

[3] H.Aznaoui, S.Raghay \& L.Aziz. "New Smart nodes distribution using Kmeans Approach to enhance Routing in WSN". Indian Journal of Science and Technology, 2016. 9.10.17485/ijst/2016/v9i46/106908.

[4] Aznaoui, H., Raghay, S., Ouakrim, Y., \& Aziz, L. "A Heuristic Algorithm of Cooperative Agents Communication for Enhanced GAF Routing Protocol in WSNs," Wireless Communications and Mobile Computing, 2019.

[5] Saroja, T. V., Ragha, L. L., \& Sharma, S. K. "Hybrid spectrum access model using game theory approach for multichannel heterogeneous mobile cognitive radio wireless sensor network," Indonesian Journal of Electrical Engineering and Computer Science, vol. 16, no. 1, pp. 116-126, 2019.

[6] Ullah, A. "Artificial bee colony algorithm used for load balancing in cloud computing," IAES International Journal of Artificial Intelligence, vol. 8, no. 2, p. 156, 2019.

[7] El Aalaoui, A., \& Hajraoui, A. "Energy efficiency of organized cluster election method in wireless sensor networks," Indonesian Journal of Electrical Engineering and Computer Science, vol. 18, no. 1, pp. 218-226, 2020.

[8] Lechani, T., Tourtchine, V., \& Amari, S. "A new modification of LEACH for efficient energy in WSN," Indonesian Journal of Electrical Engineering and Computer Science, 2020.

[9] Ullah, A., \& Nawi, N. M. "Enhancing the dynamic load balancing technique for cloud computing using HBATAABC algorithm," International Journal of Modeling, Simulation, and Scientific Computing, p. 2050041, 2020.

[10] Ullah, A., \& Nawi, N. M. "Enhancing the dynamic load balancing technique for cloud computing using HBATAABC algorithm," International Journal of Modeling, Simulation, and Scientific Computing, vol. 11, no. 05, p. 2050041, 2020.

[11] Aznaoui, H., Raghay, S., Aziz, L., Benloghfyry, A., \& Duvallet, C. "Efficient Weighted GAF routing protocol using data aggregation in WSN," 2018. 
[12] Cha SH., Lee KW. "Location Prediction for Grid-Based Geographical Routing in Vehicular Ad-Hoc Networks," In: Kim T. et al. (eds) Grid and Distributed Computing. Communications in Computer and Information Science, vol 261, Springer, Berlin, Heidelberg 2011.

[13] Chatterjee M., Das S.K., Turgut D. "WCA: A weighted clustering algorithm for mobile Ad Hoc networks," Cluster Comput. vol. 5, pp. 193-204, 2002.

[14] Chen, S.-H.; Cheng, Y.-C.; Lee, C.-H.; Wang, S.-P.; et all, "Extending sensor network lifetime via wireless charging vehicle with an efficient routing protocol," In Proceedings of the South east Con 2016, Norfolk, VA, USA, 2016.

[15] D. Goyal and M. R. Tripathy, "Routing protocols in wireless sensor networks: a survey," in Proceedings of the 2nd International Conference on Advanced Computing and Communication Technologies (ACCT '12), pp. 474-480, Rohtak, India, 2012.

[16] D. Wu, L. Bao, and R. Li, "Robust localization protocols and algorithms inwireless sensor networks using UWB," Ad-Hoc and Sensor Wireless Networks, vol. 11, no. 3-4, pp. 219-243, 2011.

[17] E. Niewiadomska-Szynkiewicz, "Localization in wireless sensor networks: Classification and evaluation of techniques," Int. J. Appl. Math. Comput. Sci., vol. 22, pp. 281-297, 2012.

[18] H. Aznaoui, S. Raghay, L. Aziz and A. Ait-Mlouk, "A comparative study of routing protocols in WSN," 2015 5th International Conference on Information \& Communication Technology and Accessibility (ICTA), Marrakech, pp. 16, 2015.

[19] H. Huang, G. Hu, and F. Yu, "Energy aware Multipath Geographic Routing for Detouring Mode in Wireless Sensor Networks," in Trans. Emerging Telecommunications Technologies, vol. 22, no. 7, pp. 375-387, 2011.

[20] Hassan, M.; Zawawi, “A. Wireless power transfer (Wireless lighting)," In Proceedings of the 2015 5th International Conference on Information \& Communication Technology and Accessibility (ICTA), Marrakech, Morocco, 2015.

[21] J. Grover, Shikha and M. Sharma, "Optimized GAF in Wireless Sensor Network," Reliability, Infocom Technologies and Optimization (ICRITO) (Trends and Future Directions), 2014 3rd International Conference on, Noida, pp. 1-6, 2014.

[22] L. Aziz, S. Raghay and A. Jamali, “A New Improved Algorithm of LEACH Protocol for Wireless Sensor Networks, “In Proceedings of 2014 Mediterranean Microwave Symposium (MMS2014), Marrakech, p. 1, 2014.

[23] L. Cheng, C. Wu, Y. Zhang, H. Wu, M. Li, and C. Maple, "A survey of localization in wireless sensor network," International Journal of Distributed Sensor Networks, vol. 2012, Article ID 962523, p. 12, 2012.

[24] O. Banimelhem, \& S. Khasawneh, "GMCAR: Grid-based multipath with congestion avoidance routing protocol in wireless sensor networks". Ad Hoc Networks, vol. 10, no. 7, pp. 1346-1361, 2012.

[25] R. Akl, P. Kadiyala, M. Haidar, "Nonuniform Grid-Based Coordinated Routing in Wireless Sensor Networks," Journal of Sensors, vol. 2009, Article ID 491349, p. 11, 2009.

[26] Razzaque, M.; Dobson, S. "Energy-Efficient Sensing in Wireless Sensor Networks Using Compressed Sensing," Sensors, vol. 14, pp. 2822-2859, 2014.

[27] Ridha Soua and Pascale Minet, "A Survey on Energy Efficient Techniques in Wireless Sensor Networks", IEEE Wireless and Mobile Networking Conference (WMNC), pp. 1-9, 2011.

[28] Sharba, M. R. R., Kadhim, H. A., Al-Abassi, S. A., \& Ali, N. S. "Online geocode in postal address using gps with synchronous database accessing," Indonesian Journal of Electrical Engineering and Computer Science, vol. 17, no. 3, pp. 1487-1492, 2020.

[29] Singh, B.P., Rajni, Singh, G. "Comparative analysis of efficient energy coverage problem of WSN with ACO and ACB-SA," Int. J. Recent Trends Eng. Technol. (IJRTET), vol. 11, no. 2, pp. 371-377, 2014. ACEEE (a subdivision of IDES)

[30] Ullah, A., Nawi, N. M., Aamir, M., Shazad, A., \& Faisal, S. N. "An Improved Multi-layer Cooperation Routing in Visual Sensor Network for Energy Minimization,"

[31] Wen, F., Wang, H., He, T., Shi, Q., Sun, Z., Zhu, M., ... \& Lee, C. "Battery-free short-range self-powered wireless sensor network (SS-WSN) using TENG based direct sensory transmission (TDST) mechanism," Nano Energy, vol. 67, p. 104266, 2020.

[32] Xiaoliang Meng, Xiaochuan Shi, Zi Wang, Shuang Wu, Chenglin Li, “A Grid-Based Reliable Routing Protocol for Wireless Sensor Networks with Randomly Distributed Clusters," Ad Hoc Networks 2016, doi: 10.1016/j.adhoc.2016.08.004

[33] Y-F Chen, X-G Fan, B Xu, "Cluster head optimization strategy for WSN based on LEACH," Comput Eng. vol. 22, no. 026, 2011.

[34] Y-h Zhu, W W-d, J Pan, T Y-p, “An energy-efficient data-gathering algorithm to prolong lifetime of wireless sensor networks," Comput. Commun. vol. 33, pp. 639-647, 2010.

[35] X. S. Li, et al., "Analysis and Simplification of Three-Dimensional Space Vector PWM for Three-Phase Four-Leg Inverters," IEEE Transactions on Industrial Electronics, vol. 58, pp. 450-464, 2011.

[36] R. Arulmozhiyal and K. Baskaran, "Implementation of a Fuzzy PI Controller for Speed Control of Induction Motors Using FPGA," Journal of Power Electronics, vol. 10, pp. 65-71, 2010.

[37] D. Zhang, et al., "Common Mode Circulating Current Control of Interleaved Three-Phase Two-Level Voltage-Source Converters with Discontinuous Space-Vector Modulation," 2009 IEEE Energy Conversion Congress and Exposition, vol. 1-6, pp. 3906-3912, 2009.

[38] Z. Yinhai, et al., "A Novel SVPWM Modulation Scheme," in Applied Power Electronics Conference and Exposition, 2009. APEC 2009. Twenty-Fourth Annual IEEE, pp. 128-131, 2009. 